www.nature.com/jhg

\title{
A commentary on high-dose enzyme replacement therapy attenuates cerebroventriculomegaly in a mouse model of mucopolysaccharidosis type II
}

\author{
Maria L Escolar \\ Journal of Human Genetics (2013) 58, 767-768; doi:10.1038/jhg.2013.115; published online 14 November 2013
}

$\mathrm{M}$ ucopolysaccharidosis type II (Hunter syndrome) is a lysosomal storage disorder characterized by a deficiency of the enzyme iduronate-2-sulfatase, which leads to the accumulation of glycosaminoglycans in visceral organs and connective tissue throughout the body. Patients with the severe form of Hunter syndrome also experience progressive neurological deterioration, which leads to severe cognitive impairment and behavioral problems. Although intravenous enzyme replacement therapy (ERT) can successfully treat many of the somatic symptoms of Hunter syndrome, it has shown little success in treating the neurological symptoms, presumably because the blood-brain barrier limits enzyme distribution to the central nervous system.

Allogeneic hematopoietic stem cell transplantation has been effective in preventing neurological deterioration in other lysosomal storage disorders; however, its role in Hunter syndrome has been controversial until recently. Bone marrow transplantation has been shown to stabilize or improve cardiovascular outcomes, hepatosplenomegaly and joint mobility in Hunter syndrome, but patients with severe disease typically show continued neuropsychological decline after treatment. ${ }^{1}$ However, a recent study in Japan evaluated 17 patients with Hunter syndrome who underwent hematopoietic stem cell transplantation using bone marrow or umbilical cord blood from 1990 to 2003 (mean follow-up, 9.6 \pm 3.5 years).

ML Escolar is at Program for the Study of Neurodevelopment in Rare Disorders, Children's Hospital of Pittsburgh, University of Pittsburgh Medical Center, Pittsburgh, PA, USA

E-mail: maria.escolar@chp.edu
The results showed stabilization or improvement of brain MRI abnormalities (brain atrophy, cribriform lesions and ventricular enlargement) in $6 / 10$ patients with the severe form of the disease. ${ }^{2}$ In addition, some patients with severe disease showed stabilized or improved ability to carry out activities of daily living (7/9 patients) and/or functional independence measure scores $(3 / 9$ patients), an indicator of physical and cognitive disability. ${ }^{2}$

Recently, a number of studies evaluating high-dose ERT in animal models of lysosomal storage disorders have shown that this approach can also reduce neuropathology and may even result in functional improvement. ${ }^{3,4}$ In previous issue of the Journal of Human Genetics, Ahn et al. ${ }^{5}$ reported that early treatment with high-dose ERT attenuated ventriculomegaly and histological abnormalities in the brains of iduronate-2sulfatase-knockout mice, suggesting its potential usefulness in treating or preventing neuropathology in patients with severe Hunter syndrome. However, as the authors noted, one of the weaknesses of their study was the lack of behavioral outcome measures. If high-dose ERT improves ventriculomegaly and even histological pathology of the brain but does not result in functional gains, then these changes in neuropathology may be not clinically relevant.

A number of studies have attempted to identify the pathological mechanisms underlying brain magnetic resonance imaging (MRI) findings in Hunter syndrome, as well as their correlation with the severity of neurological disease. Results have been conflicting, in part, because the studies were cross-sectional in design, included patients with various mucopolysaccharidosis (MPS) disorders and/ or used qualitative rather than quantitative methods. For example, in a descriptive study of patients with Hunter syndrome, Vedolin et al. ${ }^{6}$ found that cognitive impairment was associated with hydrocephalus, brain atrophy and more extensive white matter abnormalities. More recently, Manara et al. ${ }^{7}$ retrospectively analyzed 69 brain scans of 36 patients with Hunter syndrome and found that the severe phenotype correlated with white matter abnormalities, occipital hyperostosis and third ventricle enlargement (but did not distinguish between brain atrophy and hydrocephalus as the cause of ventriculomegaly). However, a prospective, longitudinal MRI volumetric study of male patients with Hunter syndrome using automated segmentation methods found that brain tissue/intracranial volume ratio and lateral ventricle/intracranial volume ratio (indicating progressive brain atrophy) correlate better with cognitive impairment than absolute lateral ventricle volume. ${ }^{8}$

Differentiating between progressive brain atrophy and communicating hydrocephalus in Hunter syndrome is difficult because these conditions show similar features on MRI. For that reason, some researchers combine the two conditions under the single term 'ventriculomegaly' in their analysis. It will be important to determine whether the ventriculomegaly observed in this study is due to atrophy or increased intracranial pressure, because improvement of intracranial pressure could lead to better cognitive outcomes, whereas improvement of brain atrophy will not. The authors may be able to answer this question by examining the histopathology specimens. 
Finally, Ahn et al..$^{5}$ did not address the issue of anti-enzyme antibodies that can decrease treatment efficacy by reducing uptake or redirection of the enzyme to target tissues. Approximately $47 \%$ of patients with Hunter syndrome in the phase II/III clinical study evaluating ERT with idursulfase developed anti-enzyme antibodies $^{9}$ as did $50 \%$ of patients in a long-term, extension study of idursulfase. ${ }^{10}$ Furthermore, $23 \%$ of patients in the extension study had neutralizing antibodies that appeared to decrease the beneficial effects of ERT on pulmonary function. No tolerization was observed by the end of the 2 -year study. ${ }^{10}$ Our group has identified early markers of brain involvement and described the natural history of neurological disease in Hunter's syndrome, ${ }^{11,12}$ as there are two groups of patients those who develop brain disease and those who do not. With more treatments available, the need to identify those who will develop brain disease before there is cognitive involvement will be very important to assure optimal outcomes.

1 Guffon, N., Bertrand, Y., Forest, I., Fouilhoux, A. \& Froissart, R. Bone marrow transplantation in children with Hunter syndrome: outcome after 7 to 17 years. J. Pediatr. 154, 733-737 (2009).

2 Tanaka, A., Okuyama, T., Suzuki, Y., Sakai, N., Takakura, H., Sawada, T. et al. Long-term efficacy of hematopoietic stem cell transplantation on brain involvement in patients with mucopolysaccharidosis type II: a nationwide survey in Japan. Mol. Genet. Metab. 107 513-520 (2012).

3 Hemsley, K. M. \& Hopwood, J. J. Emerging therapies for neurodegenerative lysosomal storage disordersfrom concept to reality. J. Inherit. Metab. Dis. 34, 1003-1012 (2011).

4 Polito, V. A., Abbondante, S., Polishchuk, R. S., Nusco, E., Salvia, R. \& Cosma, M. P. Correction of CNS defects in the MPSII mouse model via systemic enzyme replacement therapy. Hum. Mol. Genet. 19, 4871-4885 (2010)

5 Ahn, S. Y., Chang, Y. S., Sung, D. K., Ko, A.-r., Kim, C. H. Yoo, D. K. et al. High-dose enzyme replacement therapy attenuates cerebroventriculomegaly in a mouse model of mucopolysaccharidosis type II. J. Hum. Genet 58, 728-733 (2013)
6 Vedolin, L., Schwartz, I. V., Komlos, M., Schuch, A. Puga, A. C., Pinto, L. L. et al. Correlation of MR imaging and MR spectroscopy findings with cognitive impairment in mucopolysaccharidosis II. AJNR. Am. J. Neuroradiol. 28, 1029-1033 (2007).

7 Manara, R., Priante, E., Grimaldi, M., Santoro, L., Astarita, L., Barone, R. et al. Brain and spine MRI features of Hunter disease: frequency, natural evolution and response to therapy. J. Inherit. Metab. Dis. 34, 763-780 (2011).

8 Fan, Z., Styner, M., Muenzer, J., Poe, M. \& Escolar, M. Correlation of automated volumetric analysis of brain MR imaging with cognitive impairment in a natural history study of mucopolysaccharidosis II. AJNR. Am . J. Neuroradiol. 31, 1319-1323 (2010).

9 Muenzer, J., Wraith, J. E., Beck, M., Giugliani, R., Harmatz, P., Eng, C. M. et al. A phase II/III clinical study of enzyme replacement therapy with idursulfase in mucopolysaccharidosis II (Hunter syndrome). Genet. Med. 8, 465-473 (2006).

10 Muenzer, J., Beck, M., Eng, C. M., Giugliani, R., Harmatz, P., Martin, R. et al. Long-term, open-labeled extension study of idursulfase in the treatment of Hunter syndrome. Genet. Med. 13, 95-101 (2011).

11 Holt, J., Poe, M. D. \& Escolar, M. L. Early clinical markers of central nervous system involvement in mucopolysaccharidosis type II. J. Pediatr. 159, 320-326 (2011).

12 Escolar, M., Holt, J. \& Poe, M. Early clinical markers of CNS involvement in MPS II. Mol. Genet. Metab. 99, S18 (2010). 